\title{
Thrombotic thrombocytopenic purpura and membranous glomerulonephritis: A pregnancy-induced case
}

\author{
Edyta Golembiewska ${ }^{1}$, Grażyna Dutkiewicz ${ }^{1}$, Joanna Stepniewska ${ }^{1}$, Katarzyna Bobrek- \\ Lesiakowska ${ }^{1}$, Jarosław Przybyciński ${ }^{1}$, Anita Kosiarkiewicz ${ }^{2}$, Elżbieta Urasińska ${ }^{2}$, Maciej \\ Domański ${ }^{1}$, Iza Mituła ${ }^{3}$, Kazimierz Ciechanowski ${ }^{1}$, Marek Myslak ${ }^{1}$ \\ 1. Department of Nephrology, Transplantology and Internal Medicine, Pomeranian Medical University, Szczecin, Poland. 2. \\ Department of Pathology, Pomeranian Medical University, Szczecin, Poland. 3. Hemodialysis Center Avitum, Drezdenko, \\ Poland.
}

Correspondence: Edyta Golembiewska. Address: Al. Powst. WIkp. 72, 70-111 Szczecin, Poland. Email: irys@pum.edu.pl

Received: February 6, 2014

DOI: $10.5430 /$ crim.v1n2p60

Accepted: March 20, 2014

Online Published: March 25, 2014

URL: http://dx.doi.org/10.5430/crim.v1n2p60

\section{Abstract}

The association of thrombotic thrombocytopenic purpura and severe proteinuria is uncommon. The majority of such patients had already been diagnosed with systemic lupus erythematosus (SLE) and present overlapping symptoms of these two diseases. We report a case of thrombotic thrombocytopenic purpura (TTP) accompanied by severe proteinuria developed simultaneously during pregnancy. Clinical and serological data for SLE were negative. Kidney biopsy revealed features of chronic thrombotic microangiopathy and membranous glomerulonephritis.

\section{Keywords}

Membranous glomerulonephritis, Pregnancy, Thrombotic thrombocytopenic purpura

\section{Introduction}

The association of thrombotic thrombocytopenic purpura and severe proteinuria is uncommon. The majority of such patients had already been diagnosed with SLE and present overlapping symptoms of these two diseases. We report a case of thrombotic thrombocytopenic purpura (TTP) accompanied by membranous glomerulonephritis developed simultaneously during pregnancy.

\section{Case presentation}

A 29-year-old Caucasian woman, primigravida, was transferred from intensive care unit (ICU) to the Department of Nephrology, Transplantology and Internal Medicine with profound thrombocytopenia and severe proteinuria. Three weeks earlier she underwent Cesarean section in week 34 of her gestation. At the beginning HELLP syndrome was one of the suspected diagnoses, however, there was only a mild elevation of transaminases (AST $60 \mathrm{U} / \mathrm{L}$, ALT $39 \mathrm{U} / \mathrm{L}$ ).

Before pregnancy, the patient used oral contraceptives for about 10 years. 13 years before pregnancy she underwent a removal of fibroid tumor from left breast. She did not suffer from chronic illnesses. First trimester of the pregnancy was uncomplicated, in the second diabetes was diagnosed, well-controlled with diabetic diet and two injections of insulin 
daily. Since the beginning of third trimester edema of lower extremities was observed related probably to venous stasis caused by inferior vena cava compression and not to hypercoagulable state related to pregnancy. In addition, at 34 week of pregnancy, acute symptomatic thrombocytopenia, high blood pressure values (220/120 mmHg) and rapidly increasing proteinuria developed. Pregnancy was terminated necessitating a Cesarean section and a healthy infant with an Apgar score of 10 was delivered. Patient was clinically improving, but five days after delivery there was a rapid decline with hypertension crisis, vision disorders, mild speech and balance disorders. Weight gain and fluid retention were also observed. Laboratory tests showed anaemia (hemoglobin $5.1 \mathrm{mmol} / \mathrm{L}$ ), profound thrombocytopenia (19G/L), lactic dehydrogenase level $3205 \mathrm{IU} / \mathrm{L}$, total bilirubin $3 \mathrm{mg} / \mathrm{dL}$ and proteinuria in nephrotic range. Serologic test for presence of antiHBc (Hepatitis B core) antibodies was positive. Hepatits B surface antigen (HBsAg) and HBV DNA test, anti-HCV antibodies and human immunodeficiency virus (HIV) antibodies were negative. ANA antibodies were positive at a titre of 1:320. Antibodies to ds-DNA, histones, Ro/SS-A, La/SS-B, as well as antibodies to Sm and RNP were negative. A lupus anticoagulant was not detected. The titre of anti-cardiolipin antibodies was normal. PCR showed minor CMV replication 327 DNA copies/mL. Patient was transferred to ICU. Emergency CT scan of the head revealed small focal haemorrhagic lesions in cerebellum and pons. Eye examination revealed bilateral subretinal hemorrhages with retinal detachment. Peripheral blood smear showed fragmentocytes suggesting thrombotic thrombocytopenic purpura (TTP). ADAMTS13 (von Willebrand factor cleaving protease) activity was 4\% (normal range 40\%-130\%) and ADAMTS13 inhibitor level was $36 \mathrm{U} / \mathrm{mL}$ (titer of greater than $15 \mathrm{U} / \mathrm{mL}$ represents a positive anti-ADAMTS13 autoantibodies result) which confirmed the diagnosis of TTP. Treatment was initiated with methylprednisolon pulses and multiple courses of plasma exchange. As general state of the patient and biochemical parameters improved she was transferred to Department of Nephrology, Transplantology and Internal Medicine. Steroids were continued in high doses (1 g/day). Plasmapheresis was performed with the use of antihemolytic globulin. Over the next weeks an increasing proteinuria was observed (urine protein loss 25 g/day), patient required multiple units of fresh frozen plasma and albumin transfusions. RAA blockade was used (enalapril $20 \mathrm{mg}$ twice daily). Hemolytic anaemia led to several red blood cell transfusions. Additionally, intravenous immunoglobulins were introduced (Kiovig 60g each second day). By day 60, platelet count increased to $103 \mathrm{G} / \mathrm{L}$, at which point renal biopsy was done.

On light microscopy, 25 glomeruli were present. In all glomeruli double contours of glomerular basement membrane were found. In some glomeruli features of mesangiolysis and mesangial matrix expansion were present. Fresh fibrin thrombi in arterioles were not observed. Edematous endothelium was present in arteries and arterioles. Immunohistochemical staining showed deposition of IgM and C1q in mesangium and along the capillary walls, as well as C3, IgG nad IgA deposits along the capillary walls. Light microscopy showed thickening of capillaries. Endotheliosis was not observed (see Figure 1). Electron microscopy showed subepithelial deposits, effacement of podocytes foot processes and thickening of capillaries (see Figure 2 and Figure 3). Tubuloreticular inclusions were not observed. Essentially, the biopsy had typical features of both chronic thrombotic microangiopathy and membranous nephropathy.

Due to proteinuria cyclosporin was used $100 \mathrm{mg}$ twice daily. Four weeks after introduction, cyclosporin level was 80 $\mathrm{ng} / \mathrm{mL}$. Treatment of the patient was complicated by Clostridium difficile infection (treated with oral vancomycin) and urinary tract infection (Escherichia coli wild type, treated with cefuroxime intravenously).

Altogether, 27 plasmapheresis sessions were done. Steroid and cyclosporine therapy was continued in reduced doses. 18 weeks after delivery patient was discharged home with proteinuria $840 \mathrm{mg} / \mathrm{dL}$ and stable platelet count exceeding 200 G/L, serum creatinine concentration was $1.1 \mathrm{mg} / \mathrm{dL}$ (eGFR $67 \mathrm{~mL} / \mathrm{min}$ ). ADAMTS13 activity was 54\% and ADAMTS13 inhibitor level $2.5 \mathrm{U} / \mathrm{mL}$. Clinical and hematological remission of the patient’s TTP has since been maintained.

Clinical and laboratory data of the patient during observation period are presented in Table 1. 


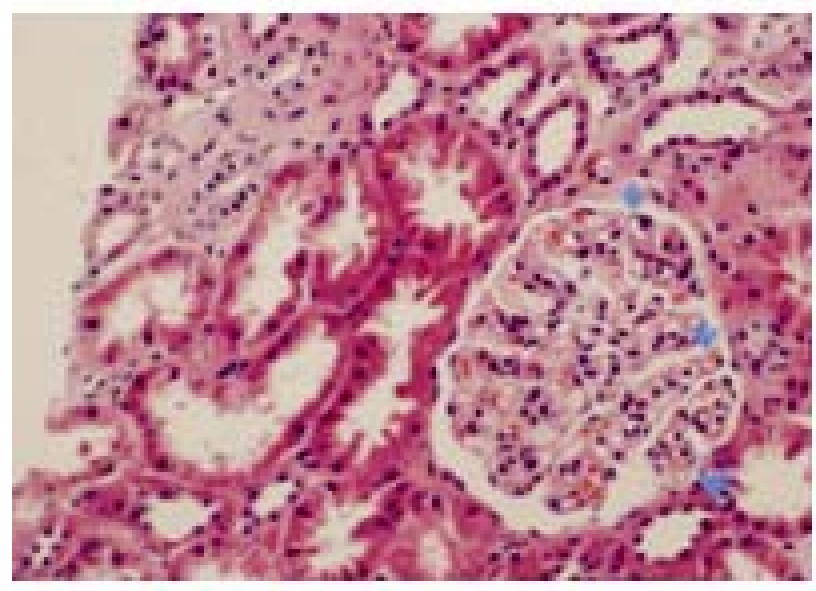

Figure 1. Light microscopy. Arrows indicate thickening of capillaries.

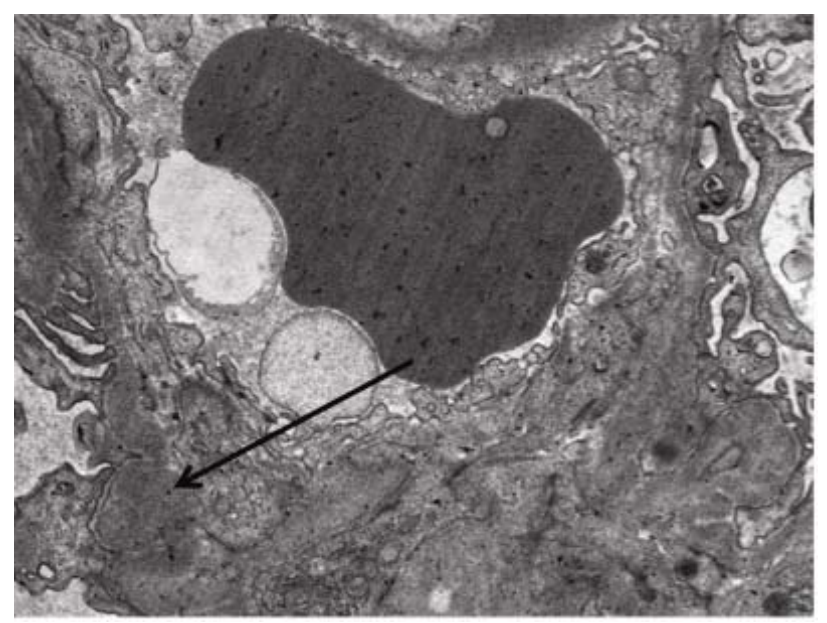

Figure 2. Electron microscopy. Focal subepithelial electron-dense deposists (arrow).

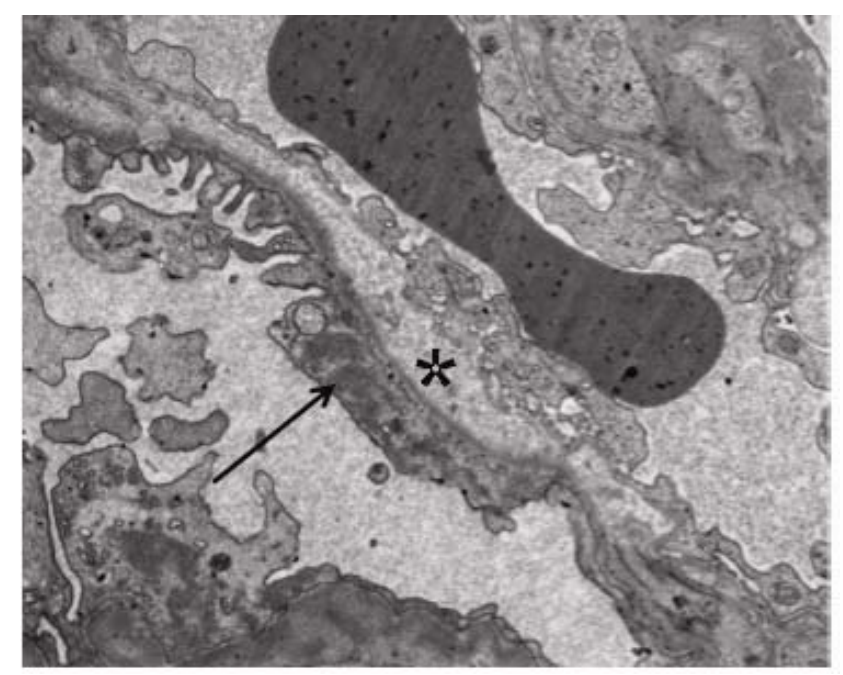

Figure 3. Electron microscopy. Focal effacement of podocytes foot processes (arrow). Thickening and double contours of capillaries (asterisk). 
Table 1. Clinical and laboratory data of the patient before delivery, after delivery, at the time of clinical worsening, at the time of biopsy, at discharge and on the day of last plasmapheresis.

\begin{tabular}{|c|c|c|c|c|c|}
\hline & $\begin{array}{c}\text { BP } \\
\text { [mmHg] }\end{array}$ & Edema & $\begin{array}{c}\text { Proteinuria } \\
\text { [g/24h] }\end{array}$ & $\begin{array}{c}\mathrm{sCr} \\
{[\mathrm{mg} / \mathrm{dl}]}\end{array}$ & CBC \\
\hline Before delivery & $220 / 120$ & +++ & 5 & ND & $\begin{array}{c}\text { WBC } 8.4[\mathrm{G} / \mathrm{l}] \\
\text { RBC } 2.6[\mathrm{~T} / \mathrm{l}] \\
\operatorname{PLT} 22[\mathrm{G} / \mathrm{l}]\end{array}$ \\
\hline After delivery & $150 / 80$ & ++ & 3 & 0.78 & $\begin{array}{c}\text { WBC } 9.0[\mathrm{G} / \mathrm{l}] \\
\text { RBC } 2.42[\mathrm{~T} / \mathrm{l}] \\
\operatorname{PLT} 22[\mathrm{G} / \mathrm{l}]\end{array}$ \\
\hline Worsening & 230/120 & +++ & 21 & 1.61 & $\begin{array}{c}\text { WBC } 5.7[\mathrm{G} / \mathrm{l}] \\
\text { RBC } 2.17[\mathrm{~T} / \mathrm{l}] \\
\text { PLT } 19[\mathrm{G} / \mathrm{l}]\end{array}$ \\
\hline Biopsy & $130 / 80$ & ++ & 25 & 0.86 & $\begin{array}{c}\text { WBC } 3.91[\mathrm{G} / \mathrm{l}] \\
\text { RBC } 3.26[\mathrm{~T} / \mathrm{l}] \\
\operatorname{PLT} 103[\mathrm{G} / \mathrm{l}]\end{array}$ \\
\hline Discharge & $155 / 90$ & + & 3.75 & 1.14 & $\begin{array}{c}\text { WBC } 7.63[\mathrm{G} / \mathrm{l}] \\
\text { RBC 3.65L [T/l] } \\
\operatorname{PLT} 106[\mathrm{G} / \mathrm{l}]\end{array}$ \\
\hline Last plasmapheresis & $110 / 60$ & - & 2.5 & 1.16 & $\begin{array}{c}\text { WBC } 8.1[\mathrm{G} / \mathrm{l}] \\
\text { RBC 3.35L [T/l] } \\
\text { PLT } 261[\mathrm{G} / \mathrm{l}]\end{array}$ \\
\hline
\end{tabular}

Note: BP blood pressure's Cr serum creatinine, CBC complete blood count, ND no data

\section{Discussion}

Thrombotic thrombocytopenic purpura is often suspected to women with a complicated pregnancy. Thrombotic thrombocytopenic purpura/hemolytic uremic syndrome (TTP/HUS) is reported mainly during pregnancy or early postpartum in $10 \%$ to $25 \%$ of patients ${ }^{[1,2]}$. The hypercoaguable state that occurs late in pregnancy and postpartum period and the progressively decreasing concentration of ADAMTS13 that appears in late pregnancy may combine to increase the risk of TTP/HUS ${ }^{[3]}$. Pregnancy may provide multiple risk factors that can also provoke acute episodes of TTP/HUS in susceptible women. Infections, drugs, cancer or surgery are frequently associated with initial episodes and relapses. Drug associated TTP/HUS may represent either an allergic or a dose related toxic reaction. It is most often reported with quinine ${ }^{[4]}$ although some cases have been reported with ticlopidine use ${ }^{[5]}$. In the reported case we considered drug induced TTP since our patient was using contraceptive drugs for about ten years. This drug association is uncertain as these drugs were discontinued several months before pregnancy, TTP did not occur during their use and also there is no evidence reported in literature for developing acquired TTP while using contraceptives.

Renal involvement is not a predominant feature of TTP, but proteinuria and hematuria in variable degrees are common symptoms. The association of TTP with primary glomerulonephritis (biopsy-proven membranous glomerulonephritis) has been described infrequently ${ }^{[6]}$. The majority of patients with TTP triggered by pregnancy and presenting proteinuria suffer from such autoimmune disorders like systemic lupus erythematosus (SLE), scleroderma or rheumatoid arthritis. In the course of these diseases renal involvement is typical but in case of reported patient no such autoimmune underlying condition was diagnosed.

Prior reports of association of TTP and severe proteinuria reaching nephrotic range include mostly patients with SLE. Clinical and serological data concerning SLE in our reported case are negative. Also, the deposits of IgM, IgA, C3 observed in the kidney biopsy, not necessarily indicate SLE. These deposits are seen in some cases of idiopathic membranous nephropathy with the incidence $35 \%, 20 \%$ and $78 \%$, respectively ${ }^{[7]}$. The majority of patients with TTP and 
SLE suffer from anemia, thrombocytopenia, fever, neurological disorders causing the diagnosis of TTP difficult due to overlapping manifestations of these two diseases. Levine and Shearn ${ }^{[8]}$ reviewed 151 cases of TTP reported before 1964 and noted that 34 (23\%) of patients had pathological findings suggesting SLE. In most cases Libman-Sachs endocarditis was the sole finding thought to indicate SLE. Nevertheless, 7 subjects (4\%) had also an evidence of proliferative glomerulonephritis. Patients with severe proteinuria may develop disturbances in blood coagulation and may be at risk for thrombotic events. The reported patient, however, had no history of prior thrombophlebitis or thrombotic events even during the long-term use of contraceptives.

In 2009 Kuppachi et al. ${ }^{[9]}$ reported a case of successful treatment with Rituximab of biopsy proven membranous nephropathy in combination with TTP. Though evidence for a direct pathogenic relationship between TTP and membranous nephropathy is lacking, it is possible that the same process that led to the plasma metalloprotease deficiency also led to immune complex deposition in the kidney.

\section{Disclosure statement}

The authors have no conflict of interest to declare.

\section{References}

[1] Ridolfi RL, Bell WR. Thrombotic thrombocytopenic purpura. Report of 25 cases and review of the literature. Medicine 1980; 60: 413-428. http://dx.doi.org/10.1097/00005792-198111000-00003

[2] Bell WR, Braine HG, Ness PM, Kickler TS. Improved survival in thrombotic thrombocytopenic purpura-hemolytic uremic syndrome. Clinical experience in 108 patients. N Engl J Med. 1991; 325: 398-403. http://dx.doi.org/10.1056/NEJM199108083250605

[3] George JN. The association of pregnancy with thrombotic thrombocytopenic purpura-hemolytic uremic syndrome. Curr Opin Hematol. 2003; 10: 339-344. http://dx.doi.org/10.1097/00062752-200309000-00003

[4] Kojouri K, Vesely SK, George JN. Quinine associated thrombotic thrombocytopenic purpura-hemolytic uremic syndrome: frequency, clinical features and long-term outcomes. Ann Intern Med. 2001; 135: 1047-1051. http://dx.doi.org/10.7326/0003-4819-135-12-200112180-00008

[5] Bennett CL, Weinberg PD, Rozenberg-Ben-Dror K, Yarnold PR, Kwaan HC, Green D. Thrombotic thrombocytopenic purpura associated with ticlopidine - a review of 60 cases. Ann Intern Med. 1998; 128: 541-544. http://dx.doi.org/10.7326/0003-4819-128-7-199804010-00004

[6] Friedlander Ma, Jacobs GH. Recurrent thrombotic thrombocytopenic purpura associated with membranous glomerulonephropathy. Am J Kidney Dis. 1991; 17: 83-85.

[7] J. Charles Jennette, Jean L. Olson, Melvin M. Schwartz, Fred G. Silva. Heptinstall's Pathology of the Kidney. Lippincott Williams \& Wilkins; Sixth edition, 2006.

[8] Levine S, Shearn MA. Thrombotic thrombocytopenic purpura and systemic lupus erythematosus. Arch Intern Med. 1964; 113: 826-836. http://dx.doi.org/10.1001/archinte.1964.00280120026006

[9] Kuppachi S, Chander P, Yoo J. Membranous nephropathy and thrombotic thrombocytopenic purpura treated with rituximab. J Nephrol. 2009; 22:561-564. 